\title{
Diets and Risk of Cancer
}

Keywords: Cancer; Drinking soda; Switch to water; Sugary soft drinks; Secondary bile acids; Diet in red meat and processed meat; The vital nutrient glutamine; Vitamin $B_{2}$ deficiency; Hibernation

\begin{abstract}
A new study is providing further evidence about the potentially life-threatening danger of drinking soda on a daily basis. Large, long-running epidemiological studies have also concluded that there is indeed a link between the foods a person eats and his risk of colon cancer. During this process, the bacteria produce compounds such as secondary bile acids that have been shown to cause inflammation and cancer in lab studies. A study by the University of Aberdeen has found first time that a higher concentration of the molecules that breakdown omega-3 fatty acids is associated with a higher chance of survival from bowel cancer. Results showed that a higher proportion of omega-3 metabolising enzyme to omega-6 metabolising enzyme is associated with less spread of the tumor and a greater chance of survival for an individual patient. Cancer stem cells can be put into hibernation by a little-known drug diphenyleneiodonium (DPI) according to researchers from the University of Salford, UK DPI effectively switches off the stem cancer cells, preventing their proliferation. It was showed that as the number of mutant KRAS copies increases, the tumor's aggressiveness and ability to metastasize also increases. Disruption of endogenous protective mechanisms determines the evolution of cancer.
\end{abstract}

\section{Introduction}

Cancer is just one of many chronic health conditions associated with sugary drink consumption, Cancer Council Victoria CEO Todd Harper said. We need more people to understand the connection and make switch to water. If you need help making the switch you can use the information materials from a registered dietitian about guide to giving up soda for good. These studies suggest that a diet in red meat and processed meat is to an increased risk of the disease [1]. In contrast, a fiber-rich diet is linked to a decreased risk. Researchers at Vanderbilt University Medical Center (VUMC) have demonstrated for the first time that it is possible to starve a tumor and stop its growth with a newly discovered small compound that blocks uptake of the vital nutrient glutamine.

\section{Stronger Case against Regular Soda Consumption}

A new study is providing further evidence about the potentially life-threatening danger of drinking soda on a daily basis. Researchers from the University of Melbourne and Cancer Council Victoria found that people who regularly drink soda have a higher risk of cancer than those who do not.

The research team studied over 35,000 Australians who had been diagnosed with an obesity-related cancer. Obesity-related cancers include kidney, pancreatic, gallbladder, and more. They found that regular soda consumption was contributing factor for many participants. The team also found that soda consumption can increase the cancer risk of people who are not overweight.

This increased cancer risk was not driven completely by obesity, said associate professor Allison Hodge, who coauthored the study.

\section{Journal of \\ Oncobiomarkers}

\author{
Robert Skopec* \\ Analyst-Researcher, Slovakia \\ Address for Correspondence \\ Robert Skopec, Analyst-Researcher, Dubnik, Slovakia, E-mail: \\ zxcbnvm7@gmail.com \\ Submission: 25 May, 2018 \\ Accepted: 06 June, 2018 \\ Published: 13 June, 2018 \\ Copyright: () 2018 Skopec R. This is an open access article distributed \\ under the Creative Commons Attribution License, which permits \\ unrestricted use, distribution, and reproduction in any medium, provided \\ the original work is properly cited.
}

Even people who were not overweight had an increased cancer risk if they regularly drank sugary soft drinks.

Alternatively, diet soda doesn't seem to present the same cancer risk because it's not as high in sugar. Though diet soda can still lead to health problems or obesity, it does not seem to have the same effect as sugary soda. As A. Hodge noted, all this suggests that the high sugar in soda is the really the key factor.

\section{Diet Can Raise or Lower Risk of Colon Cancer}

Large, long-running epidemiological studies have concluded that there is indeed a link between the foods a person eats and his risk of colon cancer, said Dr. Stephen O'Keefe, a gastroenterologist at the University of Pittsburgh School of Medicine at the American Association for Cancer Research's annual meeting. Research in recent years has shown that multitudes of bacteria that reside in the human gut-known collectively as the micobiome - could explain this connection.

Studies done in labs have shown that certain bacteria break down the remains of fatty and protein-rich foods in the large intestine. During this process, the bacteria produce compounds such as secondary bile acids that have been shown to cause inflammation and cancer in lab studies. Other bacteria, that break down fiber-rich foods in the large intestine, produce compounds such as butyrate that have been shown to be protective against cancer.

S. O'Keefe conducted a unique experiment to look at the effects of diet and colon cancer risk. His findings were published in 2015 in the journal Nature Communications.

The study included 20 African-Americans, a group that generally has very high rates of colon cancer, and 20 Africans from rural South Africa, where rates of colon cancer are very low. At the beginning of the study, the researchers analyzed the microbiomes of all the participants, and performed coloscopies to look at the health of the colon. The researchers also compared the two group's diets: The African-Americans, on average, ate two to three times more animal protein and fat than the rural Africans, and significantly less fiber.

Then, for the two weeks, the scientists asked the groups to switch diets. The rural Africans ate an American diet that was high in fat and low in fiber, including such foods as meatloaf, hamburgers, French fries, pancakes and sausage. The African-Americans, on the other 
hand, were given a high fiber, low-fat diet, with foods such as mango slices, fiber-rich cornmeal, lentils and tilapia, according to the study. At the end of the two weeks, the researchers performed the same tests once again.

There was a clear difference in just two weeks, O'Keefe said. The microbes that break down fiber were higher in abundance in the African-Americans and lower in the rural Africans - 0 before the study, the reverse was true. The compounds that these microbes produce were also increased in the African-Americans. The researchers also saw changes in how the lining of the colon looked in both groups: the lining was more inflamed in the rural Africans after they spent two weeks eating the high-fat, low-fiber diet.

O'Keefe noted that although there were changes. They all microbes and nutrients have essential role to play in the body. It was more important to look at how the microbes interact one with another. After eating the low-fat, high-fiber diet, the microbes in the African-American group showed a greater network of interaction, i.e more microbes were communicating with each other. But the interactions decreased after eating a high-fat, low-fiber diet.

Ultimately, the microbes in the gut and the compounds they produce represent a very dynamic system - and one that can be changed based on person's diet, O'Keefe said. Suffice it to say, that food has important effects on the total body [2].

\section{Omega-3s from Fish as Cancer Prevention}

Omega-3s from fish pack a stronger punch than flaxseed and other oils when it comes to cancer prevention, according to a firstever University of Guelph study. Prof. David Ma has discovered that marine-based omega- 3 s are 8 times more effective at inhibiting tumor development and growth [3]. This study is the first to compare the cancer-fighting potency of plant-versus marine-derived omega-3s on breast tumor development, said the professor in the Department of Human Health and Nutritional Sciences.

There is evidence that both omega-3s from plants and marine sources are protective against cancer. There are three types of omega-3 fatty acids: Alinoleic acid (ALA), Eicosapentaeonic acid (EPA), and Docosahexaeonic acid (DHA). ALA is plant-based and found in such edible seeds as flaxseed and in oils, such as soy, canola and hemp oil. EPA and DHA are found in marine life, such as fish, algae, and phytoplankton.

Published in the Journal of Nutritional Biochemistry, the study involved feeding the different types of omega- 3 s to mice with a highly aggressive form of human breast cancer HER-2, which affects 25 per cent of women and has a poor prognosis. Ma exposed to mice either the plant-based or the marine-based omega-3s, beginning in utero. The mice were exposed to the different omega- 3 s even before tumors developed, which allowed to compare how effective the fatty acids are in the prevention.

It's known that EPA and DHA can inhibit breast tumor growth, but no one has looked directly at how effective these omega-3s are compared to ALA. Ma found overall exposure to marine-based omega- $3 \mathrm{~s}$ reduced the size of tumors by $60-70 \%$ and the number of tumors by $30 \%$. Higher dose of the plant-based fatty acid were required to deliver the same impact as the marine-based omega-3s.
Omega-3s prevent and fight cancer by turning on genes associated with the immune system, and blocking tumor growth pathways, said Ma. It seems EPA and DHA are more effective at this. In North America, they don't get enough omega-3s from seafood, so there lies an opportunity to improve diet and help prevent the risk of breast cancer.

Based on the doses given in the study, humans should consume two to three servings of fish a week to have a same effect. Besides certain foods containing EPA and DHA, supplements and functional foods, such as omega-3 eggs or DHA milk, can offer similar cancer prevention effects.

The next step is to investigate the effects of omega- $3 \mathrm{~s}$ on other forms of breast cancer. Seeing the significant benefits omega-3s will likely be beneficial also for other types of cancer (for example, see the link between omega fatty acids and bowel cancer) [3].

\section{Link between Omega Fatty Acids and Bowel Cancer}

A study by the University of Aberdeen has found first time that a higher concentration of the molecules that breakdown omega-3 fatty acids is associated with a higher chance of survival from bowel cancer.

The study, published in the British Journal of Cancer, measured the proportion of the enzymes responsible for the metabolism of omega-3 and omega- 6 fatty acids in tumors found in bowel cancer patients, and compared it to the patient's survival.

Results showed that a higher proportion of omega-3 metabolising enzyme to omega-6 metabolising enzyme is associated with less spread of the tumor and a greater chance of survival for an individual patient. Omega- 3 and omega- 6 fatty acids are polyunsaturated fats that are thought to have opposing effects on health. This study looked specifically at the enzymes responsible for breaking down omega-3 and omega-6 fatty acids and their relationship with survival in bowel cancer.

Professor Graeme Murray who led the study explains: There is big variation in how people survive cancer of the large bowel and how they respond to treatment and we don't know what makes some people respond more favorably than others-this is what this research is trying to establish.

The molecules or metabolites that arise from the breakdown of omega-3-prevent tumor spread and we assume that with more of the enzyme that breaks down omega- 3 there will be increased metabolites of omega-3, and this will limit tumor spread. The less a tumor has spread the better the outcome. The converse is true for omega- 6 metabolising enzyme -such that higher proportion of omega-6 metabolising enzyme compared to omega- 3 could lead to worse outcome for the patient.

Prior to this study we did not know that such a relationship existed between these enzymes and survival in bowel cancer. Above findings are important because it highlights a new pathway for understanding survival from bowel cancer [4].

\section{Vitamin $B_{2}$ Deficiency Puts Cancer Cells into Hibernation}

Cancer stem cells can be put into hibernation by a little-known drug diphenyleneiodonium (DPI) according to researchers from the University of Salford, UK. DPI effectively switches off the stem cancer cells, preventing their proliferation. It's extraordinary; the 
cells just sit there as if in a state of suspended animation, explains Professor Michael Lisanti, Chair of Translational Medicine and lead investigator.

The discovery is significant because the drug halts the propagation of cancer stem cells without causing the toxic side-effects "normally" associated with more conventional chemotherapy.

Reporting their laboratory findings in the journal aging, the team observed that addition of DPI to a mixed population of cells eliminated the tumor initiating cancer stem cells. The drug was non-toxic for bulk cancer cells, which are not thought to be cancerforming. The authors describe how DPI targets more than 90 protein enzymes which feed mitochondria and help generate the cell's energy. Specifically, DPI works as an inhibitor of vitamin $\mathrm{B}_{2}$-riboflavin starving cells of energy!

DPI is selectively attacking the cancer stem cells, by effectively creating vitamin deficiency.

By turning off energy production in cancer stem cells they are creating a process of hibernation. The buty of this is that DPI makes the cancer stem cells metabolically inflexible, so they will be highly susceptible to a many other drugs.

Chemomotherapy produces many nasty side-effects, because it helps create toxic free radicals.

The Salford team - which specializes in the discovery new nontoxic therapies-and has published substantially on the anti-cancer impacts of vitamin $\mathrm{C}$ and antibiotics - is calling the discovery the start of a new type of chemotherapy, and they have a name for itmitoflavoscins. I terms of chemotherapies for cancer, we clearly need something better that what we have at present, and this is hopefully the beginning of an alternative approach to halting cancer stem cells, said Professor Federica Sotgia, a co-author of the study [5].

\section{Evolutionary Pathways of Pancreatic Cancer}

Pancreatic cancer is a form of cancer with the highest mortality in the world. Genetic changes that could explain his aggressiveness and early metastasis had not been found yet. A team at Technical University of Munich has now shown that those characteristics can be explained by specific amplifications which occur along various evolutionary pathways of the cancer. Based on this discovery, they have derived basic principles underlying the biology of pancreatic cancer [6].

Until now, scientists have failed to establish a link between properties of pancreatic cancer, such as its aggressiveness, and changes, i.e. mutations, in the tumor's genome. Pancreatic cancer forms metastases much faster than other types of cancer. The genetic causes are unclear.

A team headed by Professor Roland Rad and Professor Dieter Saur of TUM University Hospital and the German Cancer Consortium has taken an important step towards solving both mysteries [6]. With the help of various mouse models for pancreatic cancer, they have succeeded in elucidating the molecular pathways of tumor development in detail and have gained a better understanding of how various characteristics of the disease arise. The study was published in the journal Nature.
Tumor cells have multiple defective copies of a cancer gene. Healthy cells in humans possess two copies of each gene. The researchers mutated one of the two copies of the KRAS gene in mice. The gene plays a key role in cellular proliferation and is activated in $90 \%$ of all human pancreatic tumors. Such genes are referred as oncogenes. The mutant gene was often duplicated in very early stages of the cancer. In cases where a tumor had not doubled the mutated KRAS gene copy, the researchers discovered duplications in other cancer genes.

The cell amplifies the growth signal due to the presence of extra gene copies. This model of dosage amplification during tumor development had not previously been considered, says Sebastian Müller, lead author of the study. They also showed that as the number of mutant KRAS copies increases, the tumor's aggressiveness and ability to metastasize also increases. Disruption of endogenous protective mechanisms determines the evolution of cancer.

Normally, healthy cells have their own protective mechanisms to prevent mutations from accumulating. So how could the cells develop such dosage amplification without being prevented from doing so? After the KRAS mutation was induced by the researchers, other mutations in what are known as tumor suppressor genes developed. A healthy cell possesses a whole series of such protective genes to prevent cancer from developing. A significant finding by the team was that either the mutant KRAS gene or another cancer gene is amplified, depending on which tumor gene is affected and to what degree its function is impaired.

Only after the cell's inbuilt protective mechanisms have been switched off and dosage amplification occurs does a tumor ultimately form. Which pathway the cell follows, and which genes are involved then largely determine the characteristics of the pancreatic tumor.

For the first time, the dosage amplification model allows to identify genetic patterns that explain a tumor's aggressiveness and metastasis. This discovery constitutes a fundamental principle in the development of tumors and plays an essential role in other cancers. The team is now investigating the extent to which these new insights into cancer biology can be used to develop new therapeutic strategies [6].

\section{A Way to Starve Cancer}

Researchers at Vanderbilt University Medical Center (VUMC) have demonstrated for the first time that it is possible to starve a tumor and stop its growth with a newly discovered small compound that blocks uptake of the vital nutrient glutamine.

Their findings, reported in Nature Medicine, lay the groundwork for development of potential paradigm-shifting therapies targeting cancer cell metabolism that could be monitored non-invasively by positron-emission tomography (PET) imaging.

Cancer cells exhibit unique metabolic demands that distinguish them biologically from otherwise healthy cells, said Charles Manning, Ph.D., scientific director of the Vanderbilt Center for Molecular Probes and the paper's corresponding author. The metabolic specifity of cancer cells affords us rich opportunities to parlay chemistry, radiochemistry and molecular imaging to discover new cancer diagnostics as well as potential therapies. 
Glutamine is a essential amino acid for many cell functions including biosynthesis, cell signaling and protection against oxidative damage. Because cancer cells divide more rapidly than normal cells, they need more glutamine.

A protein called ACST2 is the primary transporter of glutamine into cancer cells. Elevated ACST2 levels have been linked to poor survival in many human cancers, including those of the lung, breast and colon. Genetic studies that silence ACST2 gene in cancer cells have produced dramatic anti-tumor effects.

The Vanderbilt researchers went one step further: they developed V-9302, the first highly-potent small molecule inhibitor of a glutamine transporter. In cancer cells grown in vitro and in mouse models, pharmacological blockade of ACST2 with V-9302 resulted in reduced cancer cell growth and proliferation, increased oxidative damage and increased cell death.

Targeting glutamine metabolism at the transporter level thus represents a potentially viable approach in precision medicine, the researchers concluded.

However, pairing patients with glutamine-dependent tumors and this novel class of inhibitors will require biomarkers, they cautioned. That's because response to V-9302 depends more on the activity of the ACST2 transporter than on expression of the transporter gene. Fortunately, innovative PET tracers can be developed to detect tumors by their increased rate of glutamine metabolism compared to normal surrounding tissue.

Currently five clinical trials are underway at VUMC to test the diagnostic potential of a new PET tracer called 18F-FSPG to visualize tumors of the lung, liver, ovary and colon. Manning and his CMP colleagues have also brought other tracers of glutamine metabolism, including 11C-Glutamine, into clinical trials at Vanderbilt. By attaching an imaging isotope to V-9302, Manning's group also can see if the compound is reaching its target - tumors with high rates of glutamine metabolism.

The Vanderbilt-Ingram Cancer Center is funding the development of what Manning calls this theranostic approach - the combination of therapeutics and diagnostics $[7,8]$.

\section{Conclusions}

Alternatively, diet soda doesn't seem to present the same cancer risk because it's not as high in sugar. Though diet soda can still lead to health problems or obesity, it does not seem to have the same effect as sugary soda. As A. Hodge noted, all this suggests that the high sugar in soda is the really the key factor.

Ultimately, the microbes in the gut and the compounds they produce represent a very dynamic system - and one that can be changed based on person's diet, O'Keefe said. Suffice it to say, that food has important effects on the total body [2].

Omega-3s prevent and fight cancer by turning on genes associated with the immune system, and blocking tumor growth pathways, said Ma. It seems EPA and DHA are more effective at this. In North America and in Europe too, we don't get enough omega-3s from seafood, so there is an opportunity to improve diet and help prevent the risk of breast cancer.
The molecules or metabolites that arise from the breakdown of omega-3-prevent tumor spread and we assume that with more of the enzyme that breaks down omega- 3 there will be increased metabolites of omega-3, and this will limit tumor spread.

DPI is selectively attacking the cancer stem cells, by effectively creating vitamin deficiency.

By turning off energy production in cancer stem cells they are creating a process of hibernation. For the first time, the dosage amplification model allows to identify genetic patterns that explain a tumor's aggressiveness and metastasis. This discovery constitutes a fundamental principle in the development of tumors and plays an essential role in other cancers.

The Vanderbilt researchers went one step further: they developed V-9302, the first highly-potent small molecule inhibitor of a glutamine transporter. In cancer cells grown in vitro and in mouse models, pharmacological blockade of ACST2 with V-9302 resulted in reduced cancer cell growth and proliferation, increased oxidative damage and increased cell death.

Targeting glutamine metabolism at the transporter level thus represents a potentially viable approach in precision medicine, the researchers concluded.

The Vanderbilt-Ingram Cancer Center is funding the development of what Manning calls this theranostic approach - the combination of therapeutics and diagnostics $[7,8]$.

\section{References}

1. Garcia K (2018) A new cancer study makes a stronger case against regular soda consumption. Health News.

2. Miller SG (2017) Yes, your diet can raise (or lower) your risk of colon cancer Live Science.

3. Ma D (2018) Choose omega-3s from fish over flax for cancer prevention, study finds.

4. Alnabulsi A, Swan R, Cash B, Alnabulsi A, Murray GI (2017) The differentia expression of omega- 3 and omega- 6 fatty acids metabolising enzymes in colorectal cancer and its prognostic significance. $\mathrm{Br} \mathrm{J}$ Cancer 116: 1612 1620.

5. Ozsvari B, Bonuccelli G, Sanchez-Alvarez R, Foster R, Sotgia F, et al. (2017) Targeting flavin-containing enzymes eliminates cancer stem cells (CSCs), by inhibiting mitochondrial respiration: vitamin B2 (riboflavin) in cancer therapy. Aging (Albany NY) 16: 2610-2628.

6. Rad R (2018) Insights into cancer evolution. Pancreatic cancer: gene duplication explains tumor aggressiveness.

7. Skopec R (2017) Starvation as a weapon against cancer. Int J Cancer Epidemiol Res 1: 1-5

8. Schulte ML, Fu A, Zhao P, Li J, Geng L, et al. (2018) Pharmacological blockade of ASCT2-dependent glutamine transport leads to antitumor efficacy in preclinical models. Nat Med 24: 194-202.

\section{Acknowledgement}

The author gratefully acknowledge the assistance of Dr. Marta Ballova, Ing. Konrad Balla, Livuska Ballova, and Ing. Jozef Balla. 